\title{
Covariant Graviton Propagator in Anti-de Sitter Spacetime
}

\author{
Mir Faizal \\ faizal.mir@durham.ac.uk \\ Department of Mathematics, \\ University of Durham, Durham, UK
}

September 26, 2018

\begin{abstract}
We construct the graviton propagator in $n$-dimensional anti-de Sitter spacetime in the most general covariant gauge. We then study the behaviour of this propagator for different values of the gauge parameters. We will show that in any gauge, apart from the Landau gauge, the graviton propagator in the AdS spacetime contains a complicated term involving the derivative of a hypergeometric function which can not be expressed in terms of elementary functions. We do our calculations in the Euclidean approach.
\end{abstract}

\section{Introduction}

Anti-de Sitter (AdS) spacetime is the maximally symmetric solution to the vacuum Einstein equations with a negative cosmological constant [1. It has the topology $S^{1} \times R^{n-1}$ and can be viewed as a hyperboloid in $R^{n}$. There are closed time like curves in this space. However if we unwrap the circle $S^{1}$, then no closed time-like curves are left. By AdS spacetime we will mean this unwrapped AdS spacetime, in this paper.

AdS spacetime has attracted much attention due to AdS/CFT correspondence [2]-4]. This is a correspondence between classical gravity in bulk of AdS and the quantum field theory living on its boundary. One important aspect of AdS/CFT correspondence is the calculation of correlation functions in the type II supergravity on AdS in order to study the large $\mathrm{N}$ limit of $N=4$ superconformal Yang-Mills theories [5- -7 . Bulk to Bulk propagators are required for this purpose 8 - 10. The graviton propagator in AdS spacetime in a certain Landau gauge is already known [11. The graviton propagator for $f(R)$-gravity in four dimensional AdS spacetime in a similar Landau gauge has been recently analysed [12. The Graviton propagator in AdS spacetime has also been studied in the de Donder and the Feynman gauges [13. General higher-spin quantum field theory in AdS spacetime has been thoroughly investigated in the de Donder gauge [14-[17. However, the graviton propagator in AdS spacetime has not been studied in any other gauge. In this paper we shall derive the graviton propagator in AdS spacetime in the most general covariant gauge. It may be 
noted that the covariant graviton propagator is already know for the de Sitter spacetime [18]-[19].

We shall work in the Euclidean approach of Allen and Jacobson 20. Thus we shall compute the Green's function on the $n$-dimensional hyperboloid and this will become the Feynman propagator in the AdS spacetime upon analytic continuation. So we will take the Euclidean vacuum [21] as the vacuum state for doing our calculations. We take the boundary condition as the standard boundary condition in AdS spacetime, namely that the fastest possible falloff at the boundary. Our propagator consists of three sectors namely the scalar, the vector and the tensor sectors. The tensor sector is the same as that in the work of D'Hoker, Freedman, Mathur, Matusis and Rastelli [11. The vector sector can be obtained by a trivial modification of the calculations performed for the covariant graviton propagator in de Sitter spacetime [19]. So we really need to generalize only the scalar sector of the graviton propagator in the AdS spacetime here.

\section{The field equation and the Green function}

In perturbative quantum gravity one writes the full metric in terms of a fixed background metric and small perturbations around it. We have denoted the full metric as $g_{a b}^{(f)}$ to distinguish it from the fixed background metric $g_{a b}$. We also denote the small perturbation around the fixed background metric as $h_{a b}$. So we can now write,

$$
g_{a b}^{(f)}=g_{a b}+h_{a b}
$$

This small perturbation is regarded as a field that is to be quantized. The covariant derivative along with the raising and lowering of indices is with respect to the background metric.

Thus, the action for perturbative quantum gravity in AdS spacetime can be written as

$$
S=\int d^{n} x \sqrt{-g}\left[\mathcal{L}_{\text {lin }}+\mathcal{L}_{\text {int }}\right]
$$

where $\mathcal{L}_{\text {lin }}$ is the free part of the Lagrangian which is quadratic in the field variable and $\mathcal{L}_{\text {int }}$ is part related to interactions. Here we are interested only in the free theory so we will take $\mathcal{L}_{\text {lin }}$ as our Lagrangian. This Lagrangian is invariant under the following gauge transformations,

$$
\delta_{\Lambda} h_{a b}=\nabla_{a} \Lambda_{b}+\nabla_{b} \Lambda_{a} .
$$

We have to break this gauge invariance of the theory before quantizing it. For this purpose we add the following gauge-fixing term to the original classical Lagrangian:

$$
\mathcal{L}_{\mathrm{gf}}=\frac{1}{2 \alpha}\left(\nabla_{c} h^{c b}-\frac{1+\beta}{\beta} \nabla^{b} h\right)\left(\nabla^{a} h_{a b}-\frac{1+\beta}{\beta} \nabla_{b} h\right),
$$

where $\beta$ is an non-zero arbitrary finite number.

To find the Feynman propagator, we have to first find the Euclidean Green's function for $\mathcal{L}=\mathcal{L}_{\text {lin }}+\mathcal{L}_{\text {gf }}$ and then analytically continue it back to AdS. The Euler-Lagrange field equation for this total Lagrangian is given by

$$
\frac{\partial \mathcal{L}}{\partial h_{c d}}-\nabla_{a} \frac{\partial \mathcal{L}}{\partial \nabla_{a} h_{c d}}=0
$$


This equation can be written as:

$$
\begin{aligned}
L_{a b}{ }^{c d} h_{c d}= & -\frac{1}{2} \nabla_{c} \nabla^{c} h_{a b}+\left(\frac{1}{2}-\frac{1}{2 \alpha}\right)\left(\nabla_{a} \nabla_{c} h_{b}^{c}+\nabla_{b} \nabla_{c} h_{a}^{c}\right) \\
& -\left(\frac{1}{2}-\frac{\beta+1}{\alpha \beta}\right) \nabla_{a} \nabla_{b} h-\left[\frac{(\beta+1)^{2}}{\alpha \beta^{2}}-\frac{1}{2}\right] g_{a b} \nabla_{c} \nabla^{c} h \\
& -\frac{1}{2} g_{a b}\left[1-\frac{2(1+\beta)}{\alpha \beta}\right] \nabla_{c} \nabla_{d} h^{c d}-R^{-2} h_{a b}-\frac{n-3}{2} R^{-2} g_{a b} h \\
= & 0 .
\end{aligned}
$$

Here $R$ is the radius of the hyperbolic space from which AdS spacetime is obtained.

Now we split the field $h_{a b}$ as follows:

$$
h_{b c}=A_{b c}+B_{b c}+C_{b c},
$$

where $C_{b c}, A_{a b}$ and $B_{a b}$ are the scalar, vector and tensor sectors respectively. We decompose the scalar part into a complete set of modes $C_{a b}^{\lambda \sigma}$ constructed from $\phi^{\lambda \sigma}$, where

$$
\nabla^{2} \phi^{\lambda \sigma}=-\lambda \phi^{\lambda \sigma}
$$

and $\sigma$ represents all the other labels. The modes $C_{a b}^{\lambda \sigma}$ can be written as a sum of a traceless part $W_{a b}^{\lambda \sigma}$ and the trace $X_{a b}^{\lambda \sigma}[22$,

$$
C_{a b}^{\lambda \sigma}=X_{a b}^{\lambda \sigma}+W_{a b}^{\lambda \sigma}
$$

where

$$
\begin{aligned}
X_{a b}^{\lambda \sigma} & =\frac{1}{\sqrt{n}} g_{a b} \phi^{\lambda \sigma}, \\
W_{a b}^{\lambda \sigma} & =\sqrt{\frac{n}{n-1}} \frac{1}{\sqrt{\lambda\left(\lambda+n R^{-2}\right)}}\left(\nabla_{a} \nabla_{b}+\frac{\lambda}{n} g_{a b}\right) \phi^{\lambda \sigma} .
\end{aligned}
$$

Here the factor $1 / \sqrt{n}$ and $\sqrt{n} / \sqrt{(n-1) \lambda+n R^{-2}}$ are the normalization factors. The trace of $W_{a b}^{\lambda \sigma}$ obviously vanishes. The vector part $A_{a b}$ is defined by

$$
A_{a b}=\nabla_{a} A_{b}+\nabla_{b} A_{a},
$$

where the divergence of $A_{a}$ vanishes, $\nabla^{a} A_{a}=0$, and this intern implies that the trace of $A_{a b}$ also vanishes, $g^{a b} A_{a b}=0$. The vector part can also be decomposed using a complete set of modes $A_{a b}^{\lambda \sigma}$ constructed from vector modes, $A_{a b}^{\lambda \sigma}=$ $\nabla_{a} A_{b}^{\lambda \sigma}+\nabla_{b} A_{a}^{\lambda \sigma}$, where 22 .

$$
\begin{aligned}
& \nabla^{a} A_{a}^{\lambda \sigma}=0, \\
& \nabla^{2} A_{a}^{\lambda \sigma}=\left(-\lambda+R^{-2}\right) A_{a}^{\lambda \sigma} .
\end{aligned}
$$

Both the trace and divergence of the tensor part $B_{a b}$ vanishes, $\nabla^{a} B_{a b}=g^{a b} B_{a b}=$ 0 . Thus, the tensor part can be decomposed using a complete set of tensor modes $B_{a b}^{\lambda \sigma}$, where 22

$$
\begin{aligned}
\nabla^{a} B_{a b}^{\lambda \sigma} & =0 \\
g^{a b} B_{a b}^{\lambda \sigma} & =0, \\
\nabla^{2} B_{a b}^{\lambda \sigma} & =\left(-\lambda+2 R^{-2}\right) B_{a b}^{\lambda \sigma} .
\end{aligned}
$$


Furthermore, the Green's function is given by

$$
L_{c d}^{a b} G_{a b a^{\prime} b^{\prime}}\left(x, x^{\prime}\right)=\delta_{c d a^{\prime} b^{\prime}}\left(x, x^{\prime}\right),
$$

where for any smooth function $f^{c d}(x)$, we have

$$
\int d^{n} x \sqrt{g} \delta_{c d a^{\prime} b^{\prime}}\left(x, x^{\prime}\right) f^{c d}(x)=f_{a^{\prime} b^{\prime}}\left(x^{\prime}\right) .
$$

Now we can decompose this delta function and the Green's function into scalar, vector and tensor modes as follows:

$$
\begin{aligned}
\delta_{c d a^{\prime} b^{\prime}}\left(x, x^{\prime}\right) & =\delta_{c d a^{\prime} b^{\prime}}^{(C)}\left(x, x^{\prime}\right)+\delta_{c d a^{\prime} b^{\prime}}^{(A)}\left(x, x^{\prime}\right)+\delta_{c d a^{\prime} b^{\prime}}^{(B)}\left(x, x^{\prime}\right), \\
G_{c d a^{\prime} b^{\prime}}\left(x, x^{\prime}\right) & =G_{c d a^{\prime} b^{\prime}}^{(C)}\left(x, x^{\prime}\right)+G_{c d a^{\prime} b^{\prime}}^{(A)}\left(x, x^{\prime}\right)+G_{c d a^{\prime} b^{\prime}}^{(B)}\left(x, x^{\prime}\right) .
\end{aligned}
$$

where

$$
\begin{aligned}
\delta_{c d a^{\prime} b^{\prime}}^{(C)}\left(x, x^{\prime}\right) & =\sum_{\lambda} \sum_{\sigma} C_{c d}^{\lambda \sigma}(x) C_{a^{\prime} b^{\prime}}^{* \lambda \sigma}\left(x^{\prime}\right), \\
\delta_{c d a^{\prime} b^{\prime}}^{(A)}\left(x, x^{\prime}\right) & =\sum_{\lambda} \sum_{\sigma} A_{c d}^{\lambda \sigma}(x) A_{a^{\prime} b^{\prime}}^{* \lambda \sigma}\left(x^{\prime}\right), \\
\delta_{c d a^{\prime} b^{\prime}}^{(B)}\left(x, x^{\prime}\right) & =\sum_{\lambda} \sum_{\sigma} B_{c d}^{\lambda \sigma}(x) B_{a^{\prime} b^{\prime}}^{* \lambda \sigma}\left(x^{\prime}\right),
\end{aligned}
$$

and

$$
\begin{aligned}
G_{c d a^{\prime} b^{\prime}}^{(C)}\left(x, x^{\prime}\right) & =\sum_{\lambda} \sum_{\sigma} c_{1}^{\lambda} C_{c d}^{\lambda \sigma}(x) C_{a^{\prime} b^{\prime}}^{* \lambda \sigma}\left(x^{\prime}\right), \\
G_{c d a^{\prime} b^{\prime}}^{(A)}\left(x, x^{\prime}\right) & =\sum_{\lambda} \sum_{\sigma} c_{2}^{\lambda} A_{c d}^{\lambda \sigma}(x) A_{a^{\prime} b^{\prime}}^{* \lambda \sigma}\left(x^{\prime}\right), \\
G_{c d a^{\prime} b^{\prime}}^{(B)}\left(x, x^{\prime}\right) & =\sum_{\lambda} \sum_{\sigma} c_{3}^{\lambda} B_{c d}^{\lambda \sigma}(x) B_{a^{\prime} b^{\prime}}^{* \lambda \sigma}\left(x^{\prime}\right),
\end{aligned}
$$

here the sum is a shorthand notation and includes integrals as AdS spacetime is non-compact. The constants $c_{1}^{\lambda}, c_{2}^{\lambda}, c_{3}^{\lambda}$ are determined by

$$
\begin{aligned}
& L_{c d}^{a b} G_{a b a^{\prime} b^{\prime}}^{(C)}\left(x, x^{\prime}\right)=\delta_{c d a^{\prime} b^{\prime}}^{(C)}\left(x, x^{\prime}\right), \\
& L_{c d}^{a b} G_{a b a^{\prime} b^{\prime}}^{(A)}\left(x, x^{\prime}\right)=\delta_{c d a^{\prime} b^{\prime}}^{(A)}\left(x, x^{\prime}\right), \\
& L_{c d}^{a b} G_{a b a^{\prime} b^{\prime}}^{(B)}\left(x, x^{\prime}\right)=\delta_{c d a^{\prime} b^{\prime}}^{(B)}\left(x, x^{\prime}\right) .
\end{aligned}
$$

We discuss the solutions of these equations in the next sections.

\section{Scalar Sector}

To find the total propagator, we first deal with the scalar sector. First recall that the scalar sector was decomposed using $C_{a b}^{\lambda \sigma}$, and this was expressed in terms of $X_{a b}^{\lambda \sigma}$ and $W_{a b}^{\lambda \sigma}$, where

$$
\begin{aligned}
X_{a b}^{\lambda \sigma} & =\frac{1}{\sqrt{n}} g_{a b} \phi^{\lambda \sigma}, \\
W_{a b}^{\lambda \sigma} & =\sqrt{\frac{n}{n-1}} \frac{1}{\sqrt{\lambda\left(\lambda+n R^{-2}\right)}}\left(\nabla_{a} \nabla_{b}+\frac{\lambda}{n} g_{a b}\right) \phi^{\lambda \sigma} .
\end{aligned}
$$


Furthermore, here $\lambda$ satisfies 22],

$$
\lambda \geq \frac{(n-1)^{2}}{4 R^{2}} .
$$

Now we define $K_{i j}^{\lambda}, i, j=1,2$, by

$$
\begin{aligned}
L_{a b}{ }^{c d} X_{a b}^{\lambda \sigma} & =K_{11}^{\lambda} X_{a b}^{\lambda \sigma}+K_{12}^{\lambda} W_{a b}^{\lambda \sigma}, \\
L_{a b}{ }^{c d} W_{a b}^{\lambda \sigma} & =K_{21}^{\lambda} W_{a b}^{\lambda \sigma}+K_{22}^{\lambda} W_{a b}^{\lambda \sigma} .
\end{aligned}
$$

We find that $K_{12}^{\lambda}=K_{21}^{\lambda}$. We also define $c_{i j}^{\lambda}, i, j=1,2$, by

$$
\left(\begin{array}{ll}
c_{11}^{\lambda} & c_{12}^{\lambda} \\
c_{21}^{\lambda} & c_{22}^{\lambda}
\end{array}\right)=\left(\begin{array}{ll}
K_{11}^{\lambda} & K_{12}^{\lambda} \\
K_{21}^{\lambda} & K_{22}^{\lambda}
\end{array}\right)^{-1} .
$$

We then find $c_{12}^{\lambda}=c_{21}^{\lambda}$ and

$$
\begin{aligned}
& c_{11}^{\lambda}= \frac{\beta^{2}}{n}\left\{\frac{(n-2) \alpha-2(n-1)}{n-2} \frac{1}{\lambda-(n-1) \beta R^{-2}}\right. \\
&\left.-\frac{2(n-1)}{n-2} \frac{\left[n+(n-1) \beta-\frac{n-2}{2} \alpha \beta\right] R^{-2}}{\left(\lambda-(n-1) \beta R^{-2}\right)^{2}}\right\}, \\
& \tilde{c}_{12}^{\lambda=} \frac{2 \beta}{n(n-2)} \frac{n+(n-1) \beta-\frac{n-2}{2} \alpha \beta}{\left(\lambda-(n-1) \beta R^{-2}\right)^{2}}, \\
& \tilde{c}_{22}^{\lambda}=-\frac{2}{(n-1)(n-2) R^{-4}} \frac{1}{\lambda+n R^{-2}}+\frac{\alpha}{(n-1)^{2} R^{-4}} \frac{1}{\lambda} \\
&+\frac{2(n-1)-(n-2) \alpha}{(n-2)(n-1)^{2} R^{-4}} \frac{1}{\lambda-(n-1) \beta R^{-2}} \\
&-\frac{2\left[n+(n-1) \beta-\frac{n-2}{2} \alpha \beta\right]}{(n-1)(n-2) R^{-2}} \frac{1}{\left(\lambda-(n-1) \beta R^{-2}\right)^{2}},
\end{aligned}
$$

where

$$
\begin{aligned}
\tilde{c}_{12}^{\lambda} & \equiv \frac{c_{12}^{\lambda}}{\sqrt{(n-1) \lambda\left(\lambda+n R^{-2}\right)}}, \\
\tilde{c}_{22}^{\lambda} & \equiv \frac{n c_{22}^{\lambda}}{(n-1) \lambda\left(\lambda+n R^{-2}\right)} .
\end{aligned}
$$

Define, $\Delta_{k}\left(x, x^{\prime}\right)$ to be the scalar propagator with mass $k R^{-2}$ and let $\mu\left(x, x^{\prime}\right)$ be the geodesic distance between spacelike separated points $x$ and $x^{\prime}$ in AdS spacetime and $z=\cosh ^{2}(\mu / 2 R)$, then the scalar propagator is given by [20],

$$
\Delta_{k}(z)=q_{0} z^{-a_{0}} F\left[a_{0}, a_{0}-c_{0}+1 ; a_{0}-b_{0}+1 ; z^{-1}\right],
$$

with

$$
q_{0}=\frac{\Gamma\left(a_{0}\right) \Gamma\left(a_{0}-c_{0}+1\right)}{\Gamma\left(a_{0}-b_{0}+1\right) \pi^{n / 2} 2^{n}}|R|^{2-n},
$$

where

$$
\begin{aligned}
& a_{0}=\frac{1}{2}\left[(n-1)+\sqrt{(n-1)^{2}+4 k^{2}}\right], \\
& b_{0}=\frac{1}{2}\left[(n-1)-\sqrt{(n-1)^{2}+4 k^{2}}\right], \\
& c_{0}=\frac{1}{2} n .
\end{aligned}
$$


Further, let

$$
\Delta_{k}^{(1)}\left(x, x^{\prime}\right) \equiv-\frac{1}{R^{-2}} \frac{\partial}{\partial k} \Delta_{k}\left(x, x^{\prime}\right) .
$$

Then the scalar sector of the graviton propagator in AdS, in the Euclidean approach is given by

$$
\begin{aligned}
G_{a b a^{\prime} b^{\prime}}^{(C)}\left(x, x^{\prime}\right)= & g_{a b}(x) g_{a^{\prime} b^{\prime}}\left(x^{\prime}\right) X\left(x, x^{\prime}\right) \\
& +g_{a b}(x)\left(\nabla_{a^{\prime}} \nabla_{b^{\prime}}-\frac{1}{n} g_{a^{\prime} b^{\prime}}\left(x^{\prime}\right) \nabla_{c^{\prime}} \nabla^{c^{\prime}}\right) Y\left(x, x^{\prime}\right) \\
& +g_{a^{\prime} b^{\prime}}\left(x^{\prime}\right)\left(\nabla_{a} \nabla_{b}-\frac{1}{n} g_{a b}(x) \nabla_{c} \nabla^{c}\right) Y\left(x, x^{\prime}\right) \\
& +\left(\nabla_{a} \nabla_{b}-\frac{1}{n} g_{a b} \nabla_{c} \nabla^{c}\right) \\
& \times\left(\nabla_{a^{\prime}} \nabla_{b^{\prime}}-\frac{1}{n} g_{a^{\prime} b^{\prime}} \nabla_{c^{\prime}} \nabla^{c^{\prime}}\right) Z\left(x, x^{\prime}\right)
\end{aligned}
$$

where

$$
\begin{aligned}
X\left(x, x^{\prime}\right)= & \frac{\beta^{2}}{n^{2}}\left\{\frac{(n-2) \alpha-2(n-1)}{n-2} \Delta_{-(n-1) \beta}\left(x, x^{\prime}\right)\right. \\
& -\frac{2(n-1)}{n-2}\left[n+(n-1) \beta-\frac{n-2}{2} \alpha \beta\right] \\
& \left.\times R^{-2} \Delta_{-(n-1) \beta}^{(1)}\left(x, x^{\prime}\right)\right\}, \\
Y\left(x, x^{\prime}\right)= & \frac{2 \beta}{n(n-2)}\left[n+(n-1) \beta-\frac{n-2}{2} \alpha \beta\right] \Delta_{-(n-1) \beta}^{(1)}\left(x, x^{\prime}\right), \\
Z\left(x, x^{\prime}\right)= & -\frac{2}{(n-1)(n-2) R^{-4}} \Delta_{n}\left(x, x^{\prime}\right)+\frac{\alpha}{(n-1)^{2} R^{-4}} \Delta_{0}\left(x, x^{\prime}\right) \\
& +\frac{2(n-1)-(n-2) \alpha}{(n-2)(n-1)^{2} R^{-4}} \Delta_{-(n-1) \beta}\left(x, x^{\prime}\right) \\
& -\frac{2\left[n+(n-1) \beta-\frac{n-2}{2} \alpha \beta\right]}{(n-1)(n-2) R^{-2}} \Delta_{-(n-1) \beta}^{(1)}\left(x, x^{\prime}\right) .
\end{aligned}
$$

Now after finding the graviton propagator in the most general gauge, we will discuses certain limits of this propagator. In the gauge, where $\alpha=0$ and $\beta=n /(1-n)$, we have

$$
X\left(x, x^{\prime}\right)=\frac{2}{(n-1)(n-2)} \Delta_{n}\left(x, x^{\prime}\right),
$$

and

$$
Y\left(x, x^{\prime}\right)=Z\left(x, x^{\prime}\right)=0 .
$$

So we can see that this part of the graviton propagator can be written in a simple form in the gauge when $\alpha=0$ and $\beta=n /(1-n)$. This is the gauge chosen in the work of D'Hoker, Freedman, Mathur, Matusis and Rastelli [1]. Furthermore, for $\beta=0$, we have

$$
X\left(x, x^{\prime}\right)=Y\left(x, x^{\prime}\right)=0,
$$


and

$$
\begin{aligned}
Z\left(x, x^{\prime}\right)= & -\frac{2}{(n-1)(n-2) R^{-4}} \Delta_{n}\left(x, x^{\prime}\right)+ \\
& {\left[\frac{\alpha}{(n-1)^{2} R^{-4}}+\frac{2(n-1)-(n-2) \alpha}{(n-2)(n-1)^{2} R^{-4}}\right] \Delta_{0}\left(x, x^{\prime}\right) } \\
& -\frac{2 n}{(n-1)(n-2) R^{-2}} \Delta_{0}^{(1)}\left(x, x^{\prime}\right) .
\end{aligned}
$$

Finally we take $\alpha=0$ and $\beta=n(n-2) / 4(n-1)$. For this value of $\beta$, the scalar propagator $\Delta_{-(n-1) \beta}\left(x, x^{\prime}\right)$ becomes the propagator for the conformally coupled case. Thus for $\alpha=0$ and $\beta=n(n-2) / 4(n-1)$, we have

$$
\begin{aligned}
X\left(x, x^{\prime}\right)= & \frac{(n-2)}{2(n-1)} \Delta_{m}\left(x, x^{\prime}\right)-\frac{n(n-1)(n+2)}{2(n-2)} R^{-2} \Delta_{m}^{(1)}\left(x, x^{\prime}\right) \\
Y\left(x, x^{\prime}\right)= & \frac{n(n+2)}{2(n-1)} \Delta_{m}^{(1)}\left(x, x^{\prime}\right) \\
Z\left(x, x^{\prime}\right)= & -\frac{2}{(n-1)(n-2) R^{-4}} \Delta_{n}\left(x, x^{\prime}\right)+\frac{2}{(n-2)(n-1) R^{-4}} \Delta_{m}\left(x, x^{\prime}\right) \\
& -\frac{n(n+2)}{2(n-1)(n-2) R^{-2}} \Delta_{m}^{(1)}\left(x, x^{\prime}\right)
\end{aligned}
$$

where $m=n(2-n) / 4$.

\section{Total Propagator}

To calculate the total graviton propagator we first find the Green's function for the vector part. The Green's function for the vector part of the graviton propagator in AdS spacetime is obtained by a trivial modification of the calculation's done to calculate the Green's function for the vector part of the graviton propagator in de Sitter spacetime [19. However, it will turn out that unlike Green's function for the vector part in de Sitter spacetime the Green's function for the vector part in AdS spacetime contains a very complicated term which can not simply be expressed in terms of elementary functions.

To do so we first find the equation of motion for the vector part from by substituting $h_{a b}=A_{a b}$ in Eq. (6). Thus the equation of motion for the vector part is given by

$$
\frac{1}{\alpha}\left[-\nabla^{2}+(n-1) R^{-2}\right] A_{a}=0 .
$$

Now decomposing $A_{a}$ into a complete set of vector modes and using Eq. (13), we get,

$$
\frac{1}{\alpha}\left[\lambda+(n-2) R^{-2}\right] A_{a}^{\lambda \sigma}=0 .
$$

The Green's function for this equation can be obtained by repeating the calculations that were done for obtaining the Green's function of the vector part in four dimensional de Sitter spacetime [19, for $n$ dimensional AdS spacetime. Thus the Green's function for Eq. (53), can be written as

$$
G_{a b^{\prime}}(z)=-P_{a b^{\prime}}(z)-\frac{1}{4(n-1)^{2}} \nabla_{a} \nabla_{b^{\prime}} \Delta_{0}(z) .
$$


If $n_{a}$ and $n_{b^{\prime}}$ are unit tangent vectors along the geodesics at $x$ and $x^{\prime}$ respectively, then the parallel transport $g_{a b^{\prime}}$ is defined by $g_{c^{\prime}}^{a} n_{a}(x)=-n_{c^{\prime}}\left(x^{\prime}\right)$. Now in the Allen and Jacobson formalism [20, the propagator $P_{a b^{\prime}}(z)$ is given by

$$
P_{a b^{\prime}}(z)=a(z) g_{a b^{\prime}}+b(z) n_{a} n_{b^{\prime}},
$$

where $a(z)$ and $b(z)$ again only depend on $z$ which in turn only depends on the geodesic distance between $x$ and $x^{\prime}$. They are given by

$$
\begin{aligned}
& a(z)=\left[\frac{1}{n-1} R \sinh \left(\mu R^{-1}\right) \frac{d}{d \mu}+\cosh \left(\mu R^{-1}\right)\right] \gamma(z), \\
& b(z)=\left[\frac{1}{n-1} R \sinh \left(\mu R^{-1}\right) \frac{d}{d \mu}+\cosh \left(\mu R^{-1}\right)-1\right] \gamma(z),
\end{aligned}
$$

where

$$
\gamma(z)=\left[\frac{\partial}{\partial m^{2}}\left[q_{1} F\left[a_{1}, b_{1} ; c_{1} ; z\right]\right]\right]_{\mid m^{2}=2 R^{-2}(n-1)},
$$

with

$$
q_{1}=\frac{(1-n) \Gamma\left(a_{1}\right) \Gamma\left(b_{1}\right)}{\Gamma\left(c_{1}\right) 2^{n+1} \pi^{n / 2} m^{2}} R^{-n} .
$$

Here $a_{1}, b_{1}$ and $c_{1}$ are given by

$$
\begin{aligned}
& a_{1}=\frac{1}{2}\left[(n+1)+\sqrt{(n-3)^{2}+4 m^{2} R^{2}}\right], \\
& b_{1}=\frac{1}{2}\left[(n+1)-\sqrt{(n-3)^{2}+4 m^{2} R^{2}}\right], \\
& c_{1}=\frac{1}{2} n+1 .
\end{aligned}
$$

Now by repeating the argument used to calculate the Green's function of the vector part in four dimensional de Sitter spacetime [19, for $n$ dimensional AdS spacetime, we obtain the final expression for the Green's function for the vector part of the graviton propagator in AdS spacetime,

$$
\begin{aligned}
G_{a b a^{\prime} b^{\prime}}^{(A)}\left(x, x^{\prime}\right)= & \alpha\left[\nabla_{a} \nabla_{a^{\prime}} G_{b b^{\prime}}\left(x, x^{\prime}\right)+\nabla_{b} \nabla_{a^{\prime}} G_{a b^{\prime}}\left(x, x^{\prime}\right)\right. \\
& \left.+\nabla_{a} \nabla_{b^{\prime}} G_{b a^{\prime}}\left(x, x^{\prime}\right)+\nabla_{b} \nabla_{b^{\prime}} G_{a a^{\prime}}\left(x, x^{\prime}\right)\right] .
\end{aligned}
$$

As the tensor part does not depend on the gauge parameters it will be the same in all gauge's and the full graviton propagator has already been obtained in a certain Landau gauge 11. Thus the Green's function for tensor part in the covariant gauge will be the same as that which was obtained in that Landau gauge, however we will include it here for completeness. So the Green's function for tensor part in the covariant gauge can be written as,

$$
G_{a b a^{\prime} b^{\prime}}^{(B)}\left(x, x^{\prime}\right)=\sum_{i=1}^{5} G_{i}(\mu) \mathcal{O}_{a b a^{\prime} b^{\prime}}^{i}\left(x, x^{\prime}\right),
$$

where $\mathcal{O}_{a b a^{\prime} b^{\prime}}^{i}$ are constructed from all possible linear combinations of the metrics and unit normals at $x$ and $x^{\prime}$ and along with the parallel transport. So we have,

$$
\mathcal{O}_{a b a^{\prime} b^{\prime}}^{1}=g_{a b} g_{a^{\prime} b^{\prime}},
$$




$$
\begin{aligned}
\mathcal{O}_{a b a^{\prime} b^{\prime}}^{2}= & n_{a} n_{b} n_{a^{\prime}} n_{b^{\prime}} \\
\mathcal{O}_{a b a^{\prime} b^{\prime}}^{3}= & g_{a b^{\prime}} g_{a^{\prime} b}+g_{a^{\prime} b} g_{a b^{\prime}} \\
\mathcal{O}_{a b a^{\prime} b^{\prime}}^{4}= & g_{a b} n_{a^{\prime}} n_{b^{\prime}}+n_{a} n_{b} g_{a^{\prime} b^{\prime}} \\
\mathcal{O}_{a b a^{\prime} b^{\prime}}^{5}= & g_{a b^{\prime}} n_{a^{\prime}} n_{b}+g_{a^{\prime} b} n_{a} n_{b^{\prime}} \\
& +g_{a a^{\prime}} n_{b} n_{b^{\prime}}+g_{b b^{\prime}} n_{a} n_{a^{\prime}} .
\end{aligned}
$$

The coefficient $G_{i}(z)$ also only depend on $z$ and have been explicitly calculated in terms of a single function $g(z)$ as [11,

$$
\begin{aligned}
G_{1}= & \frac{1}{n(n-2)}\left(\frac{4(z-1)^{2} z^{2}}{n+1} g^{\prime \prime}(z)+4 z(z-1)(2 z-1) g^{\prime}(z)\right. \\
& +(4 n z(z-1)+n-2) g(z)), \\
G_{2}= & -4(z-1)^{2}\left(\frac{z^{2}}{n(n+1)} g^{\prime \prime}(z)+\frac{2(n+2) z}{n(n+1)} g^{\prime}(z)+g(z)\right), \\
G_{3}= & \frac{1}{2(2-n)}\left(\frac{4(n-1) z^{2}(z-1)^{2}}{n(n+1)} g^{\prime \prime}(z)+\frac{4(n-1) z(z-1)(2 z-1)}{n} g^{\prime}(z)\right. \\
& +(4(n-1) z(z-1)+n-2) g(z)), \\
G_{4}= & -\frac{4 z(z-1)}{n(n-2)}\left(\frac{z(z-1)}{n+1} g^{\prime \prime}(z)+(2 z-1) g^{\prime}(z)+n g(z)\right), \\
G_{5}= & -\frac{x-1}{n-2}\left(\frac{2(n-1) z^{2}(z-1)}{n(n+1)} g^{\prime \prime}(z)+\frac{z(4(n-1) z-3 n+4)}{n} g^{\prime}(z)\right. \\
& +(2(n-1) z-n+2) g(z)),
\end{aligned}
$$

where $g(z)$ is given by

$$
g(z)=g_{0}(z)+g_{1}(z)+g_{2}(z)+g_{3}(z)
$$

with

$$
\begin{aligned}
g_{0}(z)= & \frac{n(n+1) \Gamma((n-2) / 2)}{(n-1) 2^{n+2} \pi^{n / 2}} \frac{d}{d z}\left[\frac{\int_{0}^{z} d z^{\prime} F\left[-n+2,1 ;(4-n) / 2 ; z^{\prime}\right]}{[z(z-1)]^{n / 2}}\right], \\
g_{1}(z)= & -\frac{n(n+1) \Gamma((n+2) / 2)}{2^{n+2} \pi^{n / 2}(n-2)(n-1) n} \frac{4(n-1) z(z-1)+n}{[z(z-1)]^{(n+2) / 2}}, \\
g_{3}(z)= & \frac{n(n+1) \Gamma((n+2) / 2)}{(n-2)(n-1) 2^{n+2} \pi^{n / 2}} \frac{2 z-1}{[z(z-1)]^{(n+2) / 2}}, \\
g_{4}(z)= & (-1)^{(n-1) / 2} \frac{\Gamma((n+3) / 2) n}{(n+2)(n-1)^{2} \pi^{(n-1) / 2}} \\
& \times F[2, n+1 ;(n+4) / 2 ; 1-z] .
\end{aligned}
$$

Thus, the total graviton propagator in AdS spacetime is given by the sum of the scalar, vector and tensor parts of the graviton propagator. We can now write the graviton propagator, in AdS spacetime, in the most general covariant gauge as:

$$
G_{a b a^{\prime} b^{\prime}}=G_{a b a^{\prime} b^{\prime}}^{(C)}\left(x, x^{\prime}\right)+G_{a b a^{\prime} b^{\prime}}^{(A)}\left(x, x^{\prime}\right)+G_{a b a^{\prime} b^{\prime}}^{(B)}\left(x, x^{\prime}\right),
$$


where the scalar contribution to the graviton propagator $G_{a b a^{\prime} b^{\prime}}^{(C)}\left(x, x^{\prime}\right)$ is given by Eq. (43), the vector contribution to the graviton propagator $G_{a b a^{\prime} b^{\prime}}^{(A)}\left(x, x^{\prime}\right)$ is given by Eq. (60), and the tensor contribution to the graviton propagator $G_{a b a^{\prime} b^{\prime}}^{(B)}\left(x, x^{\prime}\right)$ is given by Eq. (61).

\section{Conclusion}

In this paper we have derived the graviton propagator in the most general covariant gauge. This propagator can be used to calculate the correlation functions in the Bulk of AdS spacetime, which in turn can be used to study certain aspects of the AdS/CFT correspondence. It may be noted the the graviton propagator in AdS spacetime has already been derived in a certain Landau gauge [11, and used to study certain aspects of the AdS/CFT correspondence [23]-[27.

We have also seen in this paper that in any gauge, apart from the Landau gauge, the graviton propagator in the AdS spacetime has a very complicated form. This is because the the vector part of the graviton propagator in the AdS spacetime contains a derivative of the hypergeometric function which can not be simply expressed in terms of elementary functions. As the vector part of the graviton propagator is proportional to the gauge parameter $\alpha$, it vanishes in the Landau gauge. It may be noted that in de Sitter spacetime, this problem does not arise and it is possible to write the derivative of the hypergeometric function in terms of elementary functions, at least in four dimentions [18-19. As in the work of D'Hoker, Freedman, Mathur, Matusis and Rastelli [11, a certain Landau gauge was chosen, the AdS graviton propagator they derived did not contain this complicated term and thus could be easily written in terms of elementary functions.

\section{Acknowledgement}

I would like to thank Atsushi Higuchi for useful discussions.

\section{References}

[1] S. W. Hawking and G. F. R. Ellis, The Large Scale Structure of SpaceTime. Cambridge University Press (1973)

[2] J. Maldacena, Adv. Theor. Math. Phys. 2, 231 (1998)

[3] S. S. Gubser, I. R. Klebanov and A. M. Polyakov, Phys. Lett. B428, 105 (1998)

[4] E. Witten, Adv. Theor. Math. Phys. 2, 253 (1998)

[5] M. Giordano and R. Peschanski, JHEP. 037, 037 (2010)

[6] K. Okuyama, JHEP. 036, 0802 (2008)

[7] P. M. Chesler and L. G. Yaffe, Phys. Rev. D78, 045013 (2008)

[8] D. Z. Freedman, S. D. Mathur, A. Matusis and L. Rastelli, Phys. Lett. B452, 61 (1999) 
[9] E. D'Hoker and D. Z. Freedman, Nucl. Phys. B544, 612 (1999)

[10] H. Liu, Phys. Rev. D60, 106005 (1999)

[11] E. D'Hoker, D. Z. Freedman, S. D. Mathur, A. Matusis and L Rastelli, Nucl. Phys. B562, 330 (1999)

[12] Y. S. Myung, Eur. Phys. J. C71, 1550 (2011)

[13] R. Manvelyan, K. Mkrtchyan and W. Ruehl, Nucl. Phys. B803, 405 (2008)

[14] R. Manvelyan and W. Ruhl, Nucl. Phys. B751, 285 (2006)

[15] R. Manvelyan and W. Ruhl, Nucl. Phys. B796, 457 (2008)

[16] R. Manvelyan and W. Ruhl, Nucl. Phys. B733, 104 (2006)

[17] R. Manvelyan and W. Ruhl, Nucl. Phys. B797, 371 (2008)

[18] A. Higuchi and S. S. Kouris, Class. Quant. Grav. 18, 4317 (2001)

[19] B. Allen and M. Turyn, Nucl. Phys. B292, 813 (1987)

[20] B. Allen and T. Jacobson, Commun. Math. Phys. 103, 669 (1986)

[21] G. Gibbons and S. W. Hawking, Phys. Rev. D15, 2738 (1977)

[22] R. Camporesi and A. Higuchi, J. Math. Phys. 35, 4217 (1994)

[23] O. Aharony, A. B. Clark, and A. Karch, Phys. Rev. D74, 086006 (2006)

[24] G. Chalmers and J. Erdmenger, Nucl. Phys. B585, 517 (2000)

[25] E. D'Hoker, D. Z. Freedman, S. D. Mathur, A. Matusis and L. Rastelli, Nucl. Phys. B562, 353 (1999)

[26] E. D'Hoker, D. Z. Freedman and L. Rastelli, Nucl. Phys. B562, 395 (1999)

[27] E. D'Hoker, S. D. Mathur, A. Matusis and L. Rastelli, Nucl. Phys. B589, $38(2000)$ 